\title{
BART inhibits pancreatic cancer cell invasion by inhibiting ARL2-mediated RhoA inactivation
}

\author{
KEISUKE TANIUCHI, SHINJI IWASAKI and TOSHIJI SAIBARA \\ Department of Gastroenterology and Hepatology, Kochi University Medical School, Nankoku, Kochi 783-8505, Japan
}

Received May 2,2011; Accepted June 10, 2011

DOI: 10.3892/ijo.2011.1156

\begin{abstract}
We report that BART plays a role in inhibiting cell invasion by regulating the activity of the Rho GTPase protein RhoA in pancreatic cancer cells. BART was originally identified as a binding partner of ARL2, a small G-protein implicated as a regulator of microtubule dynamics and folding. We show that BART interacts with GTP-bound ARL2 and is required for the binding of GTP-bound ARL2 with active forms of RhoA at leading edges in migrating cancer cells. GTP-bound ARL2 inactivates RhoA and BART prevents ARL2 from regulating RhoA activity. Thus, BART binds to and functions as an inhibitor of ARL2 at leading edges of migrating cells, thereby increasing the amount of active RhoA. Treatment with the Rho inhibitor $\mathrm{C} 3$ exoenzyme induces cell invasion by pancreatic cancer cells to the same level as that of cells in which BART is stably knocked down by RNA interference. GTP-bound ARL2 acts as a RhoA inhibitor by a mechanism that involves the induction of actin-cytoskeleton rearrangements. We show that BART decreases actin-cytoskeleton rearrangements by inhibiting ARL2 function and by increasing the amount of active RhoA in pancreatic cancer cells. Our results imply that BART increases active RhoA by inhibiting ARL2 function, which in turn inhibits invasiveness of cancer cells.
\end{abstract}

\section{Introduction}

Binder of Arl Two (BART) is a soluble 19-kDa protein that was originally purified from bovine brain and identified as a binding partner of small G-protein ADP-ribosylation factorlike 2 (ARL2) (1). ARL proteins lack the biochemical and genetic activities characteristic of the ADP-ribosylation factor

Correspondence to: Dr Keisuke Taniuchi, Department of Gastroenterology and Hepatology, Kochi University Medical School, Nankoku, Kochi 783-8505, Japan

E-mail: jm-ktaniuchi@kochi-u.ac.jp

Abbreviations: PDAC, pancreatic ductal adenocarcinoma; siRNA, small interfering RNA; RhoGAP, Rho GTPase-activating proteins

Key words: small G-protein, Rho GTPase, pancreatic cancer, cell invasion, actin-cytoskeleton
(ARF) family, despite the 40-60\% identity between ARF and ARL proteins (2). ARL2 has been implicated as a regulator of microtubule dynamics and folding (3), but its function remains largely unknown. Nuclear magnetic resonance analysis recently showed that BART forms a novel fold composed of six $\alpha$-helices that form three interlocking L shapes (4). GTP-ARL2 assumes a typical small GTPase fold with a unique N-terminal $\alpha$-helix conformation (5). The regions involved in the binding of ARL2 to its protein partners localize to these loop regions (4). The interactions between ARL2 and BART involve two interfaces: a conserved N-terminal LLXIL motif of ARL2 embedded in a hydrophobic cleft of BART, and the switch regions of ARL2 that interact with helix $\alpha 3$ of BART (5). This novel partner recognition and binding mode is different from that of other small GTPase-effector interactions and provides the molecular basis for the high specificity of ARL2-BART binding (5).

BART interaction with ARL2 affects the transcriptional activity and nuclear retention of signal transducer and activator of transcription 3 (STAT3), which is both a cytoplasmic signaling molecule and a nuclear transcription factor (6). Recent studies have linked STAT3 to the metastatic progression of several different cancer types. Studies using mouse embryo fibroblasts established STAT3 as a component of the Rho GTPase-signaling cascade $(7,8)$. Rho GTPases are important regulators of both actin dynamics and cell-substratum adhesions in migratory cells, and are therefore critical in the process of tumor invasion and angiogenesis (9). Although the mechanisms that contribute to the constitutive activation of STAT3 in cancer invasion and metastasis are currently unclear, the binding of BART to ARL2 might contribute to the function of cell migration through the Rho GTPase-signaling cascade.

We previously reported that regulation of BART posttranscriptional modification via intracellular CD24 binding to G3BP in stress granules contributes to inhibition of invasion and metastasis of pancreatic ductal adenocarcinoma (PDAC) cells (10). In this study, we report the mechanism by which BART regulates RhoA activity through its binding to ARL2 in PDAC cells. GTP-bound ARL2 directly binds to active forms of RhoA and plays a role in inhibiting activation of RhoA. BART forms a complex with ARL2 and RhoA, resulting in the promotion of GTP-RhoA loading in PDAC cells. Consequently, BART prevents ARL2 from regulating RhoA activity and thereby inhibits cell invasion by restricting surface rearrangements of the actin cytoskeleton. 


\section{Materials and methods}

Cell culture. The human PDAC cell line S2-013, a subline of SUIT-2, was obtained from Dr T. Iwamura (Miyazaki Medical College, Miyazaki, Japan) (11). The human PDAC cell line PANC-1 was obtained from the ATCC. Cells were grown in Dulbecco's modified Eagle's medium (DMEM; Gibco-BRL, Carlsbad, CA) supplemented with $10 \%$ heat-inactivated fetal calf serum (FCS) at $37^{\circ} \mathrm{C}$ saturated with $5 \% \mathrm{CO}_{2}$ in a humid atmosphere.

Antibodies. Rabbit anti-BART antibody (MGC1121) was purchased from ProteinTech (Chicago, IL). Goat anti-ARL2 antibody (IMG-3515) was obtained from Imgenex (San Diego, CA). Monoclonal antibodies against RhoA (26C4), myc (9E10), and rabbit anti-myc antibody (A14) were purchased from Santa Cruz Biotechnology (Santa Cruz, CA). Monoclonal antibody against Rac1 (610650) was obtained from BD Transduction Laboratory (Palo Alto, CA).

In vivo binding of BART with ARL2. The entire cDNA coding sequence of wild-type ARL2 was amplified by RT-PCR and the product was inserted into pcDNA3.1(+)/myc-His A (Invitrogen, Carlsbad, CA). The myc-tagged ARL2 construct was transiently transfected into S2-013 cells using FuGENE6 (Roche, Penzberg, Germany), according to the manufacturer's instructions. At $48 \mathrm{~h}$ after transfection, the cells were lysed in lysis buffer [20 mM HEPES (pH 7.4), $100 \mathrm{mM} \mathrm{KCl,} 5 \mathrm{mM}$ $\mathrm{MgCl}_{2}, 0.5 \%$ Triton X-100, protease inhibitor cocktail tablets (Roche)] and immunoprecipitated with $2 \mu \mathrm{g}$ of anti-myc or anti-BART antibody. The interaction of myc-ARL2 with endogenous BART as an immune complex was analyzed by Western blotting with anti-myc and anti-BART antibodies.

Confocal immunofluorescence microscopy. Cells were fixed with $4 \%$ paraformaldehyde, permeabilized with $0.1 \%$ Triton $\mathrm{X}-100$, covered with blocking solution (3\% BSA/PBS), and then incubated with the primary antibody for $1 \mathrm{~h}$. Alexa 488 , Alexa 546, Alexa 594 and Alexa 633-conjugated secondary antibodies (Molecular Probes, Carlsbad, CA) were used with or without rhodamine phalloidin (Cytoskeleton, Denver, CO). Each specimen was visualized with a Zeiss LSM 510 META microscope (Carl Zeiss, Gottingen, Germany).

Affinity precipitation of BART and RhoA with GST-ARL2. The entire cDNA coding sequence of wild-type ARL2 was inserted into the pGEX-6P1 vector (GE Healthcare, Piscataway, NJ). The GST-ARL2 fusion protein was produced in Escherichia coli, purified with glutathione-Sepharose beads, and used for affinity precipitation in a GST-pull-down assay. S2-013 cells were lysed in lysis buffer and equal amounts of total lysate were incubated with $8 \mu \mathrm{g}$ of the GST-ARL2 fusion protein at $4^{\circ} \mathrm{C}$ for $1 \mathrm{~h}$. GST was used as a control. Western blotting was performed to detect co-precipitated BART and RhoA using anti-BART and anti-RhoA antibodies, respectively.

Wound healing immunostaining assay. Cells were transfected with the indicated plasmids using FuGENE6. At $48 \mathrm{~h}$ after transfection, a wound in the form of a cross was made through the confluent cell monolayer with a plastic pipette tip and the cells were then allowed to polarize and migrate toward the wound. After $4 \mathrm{~h}$, the cells were immunostained with the primary antibody and then incubated with fluorophore-conjugated secondary antibodies as described above. Each specimen was visualized with a Zeiss LSM 510 META microscope.

Affinity precipitation using a GST-bound RhoA interactive binding domain. The pGEX-6P1 plasmids encoding $\mathrm{p} 21-$ activated kinase (PAK)-Cdc42/Rac interactive binding (CRIB) domain or Rhotekin were kindly provided by Dr K. Johnson (University of Nebraska Medical Center, Omaha, NE). These GST fusion proteins were purified with glutathione-Sepharose beads and used for affinity precipitation in GST-pull-down assays to estimate the activity of Racl and RhoA. Cells were transfected with the indicated plasmids using FuGENE6. At $48 \mathrm{~h}$ after transfection, equal amounts of protein from each cell lysate were incubated with $8 \mu \mathrm{g}$ of GST-fusion protein, and the bound proteins were detected by Western blotting using anti-Rac1 or anti-RhoA antibodies.

Small interfering RNA-expressing constructs and the generation of stable cell lines. Small interfering RNA-methodologies were performed as previously reported (10).

Inhibition of endogenous RhoA by the Rho inhibitor C3 exoenzyme. The Rho inhibitor $\mathrm{C} 3$ exoenzyme from Clostridium botulinum (Calbiochem, San Diego, CA) was introduced into cells using FuGENE6. Briefly, $20 \mu \mathrm{g} / \mathrm{ml} \mathrm{C} 3$ exoenzyme was mixed with FuGENE6 in medium lacking FCS and incubated with cells for $14 \mathrm{~h}$ at $37^{\circ} \mathrm{C}$ before GST-Rhotekin pull-down and Matrigel invasion assays were performed, as described below.

Matrigel invasion assay. A two-chamber invasion assay was used to assess cell invasion (24-well plates, $8 \mu \mathrm{m}$ pore size, membrane coated with a layer of Matrigel extracellular matrix proteins; Becton-Dickinson, San Jose, CA). First, 4.0x $10^{4}$ cells were seeded in serum-free medium into the upper chamber and allowed to invade toward 5\% FCS as a chemoattractant in the lower chamber. After $20 \mathrm{~h}$ of incubation, the number of invading cells at the bottom of the membrane was estimated by counting three independent visual fields under a microscope.

BART-rescue construct. The entire coding sequence of BART cDNA was amplified by RT-PCR. The product was subsequently inserted into the pCMV6 Entry vector (Origene, Rockville, MD) bearing a C-terminal myc-DDK-tag. This BART-rescue construct was transiently transfected into cells using FuGENE6 (Roche).

Immunoprecipitation. S2-013 cells were transfected with the indicated myc-tagged plasmids using FuGENE6. After $48 \mathrm{~h}$, the cells were lysed in lysis buffer and equal amounts of cell lysate were incubated with $2 \mu \mathrm{g}$ of the indicated antibody or control IgG monoclonal antibody (isotype control) and protein G Sepharose. Co-immunoprecipitated proteins were detected by Western blotting using the indicated antibodies.

GST-mutant ARL2 binding assay in vitro. The $\mathrm{pET} 23 \mathrm{~b}$ plasmids encoding ARL2-Q70L or ARL2-T30N, with the indicated ARL2 amino acid substitutions (numbers refer to the corre- 
A

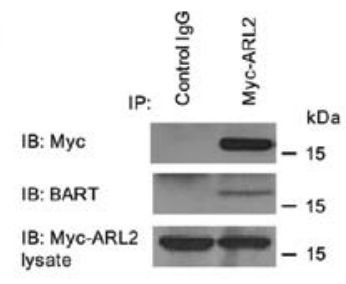

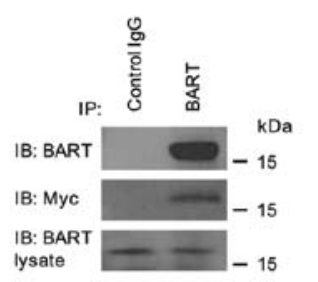

B

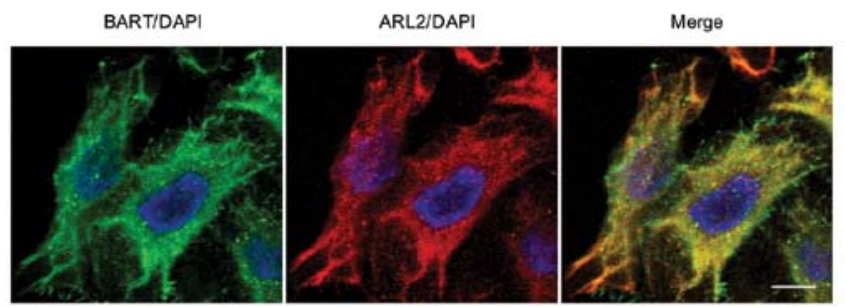

C

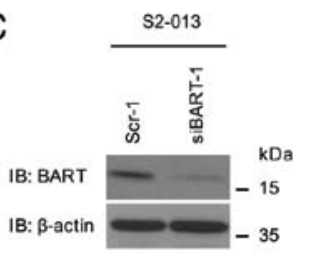

D
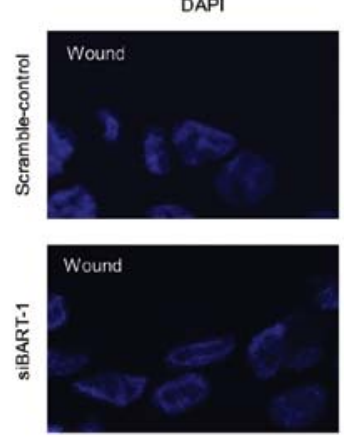
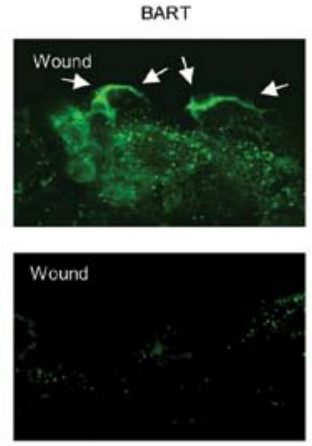
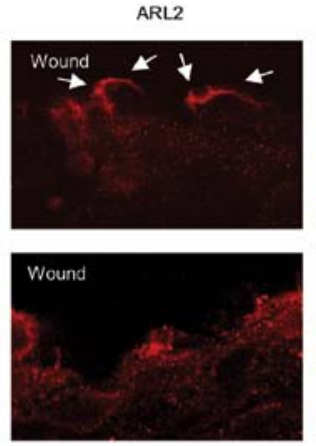
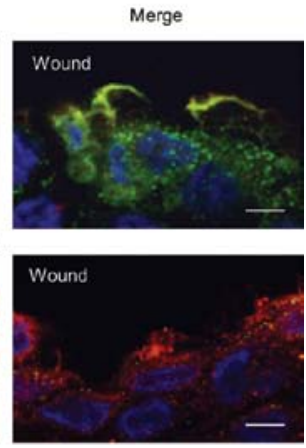

Figure 1. BART binds to ARL2 at the leading edges of migrating cells. (A) Immunoprecipitation of endogenous BART or exogenous ARL2 from S2-013 cells. A myc-tagged ARL2 construct was transiently transfected into S2-013 cells and immunoprecipitated with anti-myc or anti-BART antibodies. Immunoprecipitates were examined by Western blotting using anti-BART and anti-myc antibodies. Rabbit IgG was used as an isotype control. (B) Immunocytochemical staining of S2-013 cells using anti-BART (green) and anti-ARL2 (red) antibodies. Blue, DAPI staining. Bar, $10 \mu \mathrm{m}$. (C) Western blot analysis using the anti-BART antibody showing one representative clone of S2-013 cells transfected with siRNA for BART (siBART-1) as compared to a scrambled control clone (Scr-1). (D) Confluent scramble-control (upper panels) and BART RNAi S2-013 cells (lower panels) were wounded. After 4 h, the cells were immunostained with anti-BART (green) and anti-ARL2 (red) antibodies. Blue, DAPI staining. Arrows, colocalized BART and ARL2 at the leading edge. Bars, $10 \mu \mathrm{m}$.

sponding amino acids in ARL2), were kindly provided by $\mathrm{Dr}$ N. Cowan (New York University Medical Center, New York, NY) (12). GST-ARL2-Q70L and GST-ARL2-T30N were constructed by subcloning mutant ARL2 from the pET23b plasmids into pGEX-6P1 and subsequent confirmation of the DNA sequence. GST-ARL2-Q70L and T30N fusion proteins were produced in $E$. coli and purified as described above. Six micrograms of His-tagged wild-type RhoA protein (Cytoskeleton) was incubated with either GTP $\gamma \mathrm{S}$ or with GDP at a final concentration of $1 \mathrm{mM}$ in $20 \mu \mathrm{l}$ of reaction buffer [50 mM HEPES (pH 7.4), $100 \mathrm{mM} \mathrm{NaCl}, 10 \mathrm{mM} \mathrm{MgCl}_{2}$, $5 \mathrm{mM}$ EDTA, $1 \mathrm{mM}$ DTT] at $30^{\circ} \mathrm{C}$ for $15 \mathrm{~min}$. The concentration of $\mathrm{MgCl}_{2}$ was then increased to $50 \mathrm{mM} \mathrm{MgCl}$, and $8 \mu \mathrm{g}$ of GST-tagged Q70L or T30N was added and incubated at $4^{\circ} \mathrm{C}$ for $4 \mathrm{~h}$. GST was used as a control. After the beads were extensively washed with lysis buffer, bound RhoA was detected by Western blotting using an anti-RhoA antibody.

In vitro Rho-GAP assay. Wild-type ARL2 that was fused to GST as described above, was released by cleavage with factor $\mathrm{Xa}$ as previously reported (13). In vitro GAP assays were performed in triplicate using the RhoGAP Assay Biochem Kit (Cytoskeleton). His-tagged RhoA (5 $\mu \mathrm{g})$ was incubated with wild-type ARL2 protein $(8 \mu \mathrm{g})$ and $200 \mu \mathrm{M}$ GTP with or without p50 RhoGAP protein $(8 \mu \mathrm{g})$ at $37^{\circ} \mathrm{C}$ for $20 \mathrm{~min}$ in a reaction mixture $(20 \mu \mathrm{l})$ containing $25 \mathrm{mM}$ HEPES ( $\mathrm{pH} 7.4)$, $100 \mathrm{mM} \mathrm{NaCl}, 2 \mathrm{mM} \mathrm{MgCl}$ and $1 \mathrm{mM}$ DTT. Phosphate generated by hydrolysis of GTP was measured by adding CytoPhos reagent (Cytoskeleton) and the absorbance was read at $650 \mathrm{~nm}$.

Statistical analysis. The significance of differences between groups was determined using Student's t-test, the Mann-Whitney $\mathrm{U}$ test or Fisher's exact test, as appropriate. $\mathrm{P}<0.05$ was considered statistically significant.

\section{Results}

Colocalization of BART and ARL2 in PDAC cells. To confirm the interaction of BART and ARL2 in PDAC cells, we performed co-immunoprecipitation experiments. The myctagged ARL2 construct was transfected into human PDAC S2-013 cells, and exogenous ARL2 and endogenous BART were immunoprecipitated using anti-myc and anti-BART antibodies, respectively. Immunoblotting of the precipitates revealed that myc-ARL2 co-immunoprecipitated with endogenous BART (Fig. 1A). We next examined the subcellular 
A

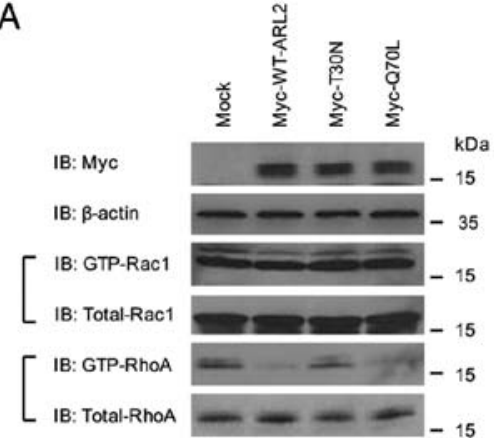

C

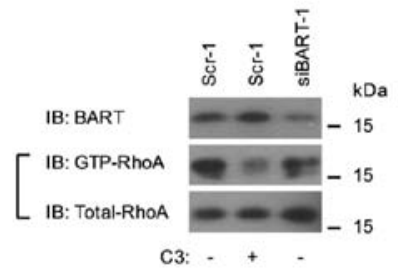

$\mathrm{E}$
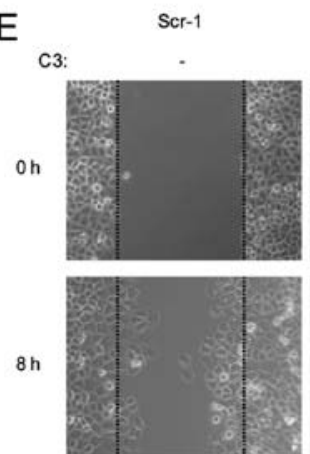
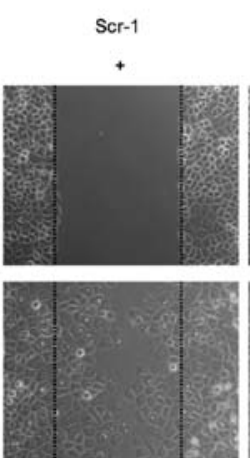

B

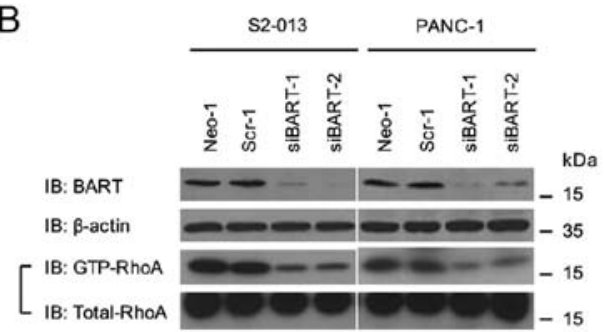

\footnotetext{
D
}
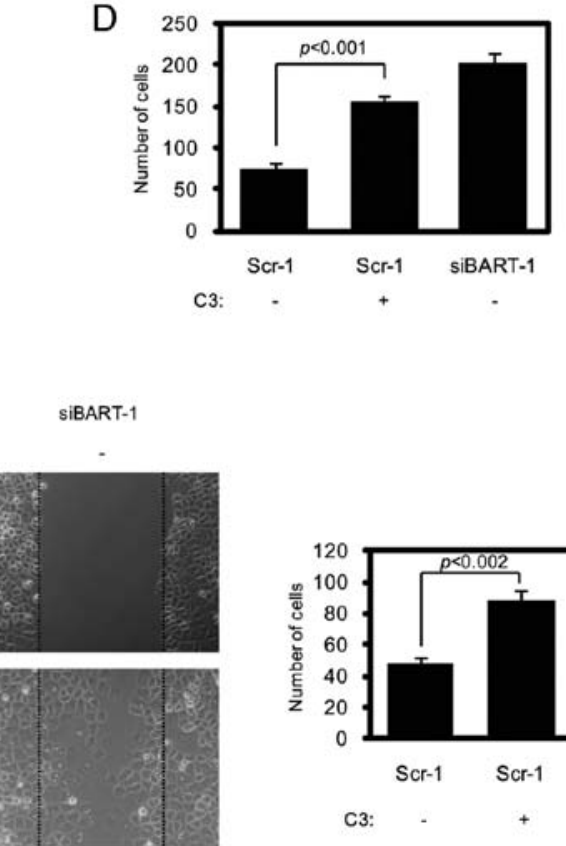

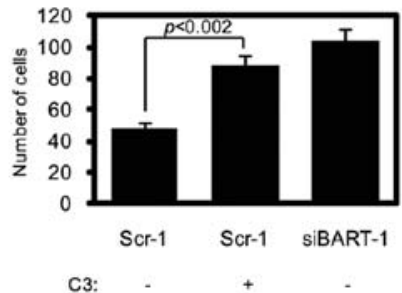

Figure 2. BART and GTP-ARL2 modulates RhoA activity, and RhoA is associated with cell invasion. (A) Myc-tagged wild-type ARL2 or its point mutants Q70L and T30N were transiently transfected into S2-013 cells and the amount of active, GTP-loaded Rac1 and RhoA were determined by GST-pull-down assays using GST-PAK-CRIB and GST-Rhotekin, respectively. Precipitates were examined by Western blotting using anti-Rac1 and anti-RhoA antibodies. (B) Western blotting with the anti-BART antibody showing each two representative clones (siBART-1 and 2) of S2-013 and PANC-1 cells transfected with BART siRNA as compared to mock (Neo-1) and scrambled (Scr-1) control clones. The amount of active, GTP-loaded RhoA in the control and BART RNAi cells was determined using GST-pull-down assays with GST-Rhotekin. Precipitants were examined by Western blotting using anti-RhoA antibody. (C) Scramble-control cells of S2-013 were preloaded with or without C3 exoenzyme, and active RhoA was assessed by GST-pull-down assays using GST-Rhotekin, followed by Western blotting. (D) Scramble-control S2-013 cells pretreated with or without C3 exoenzyme and non-treated silenced BART S2-013 cells were plated on Matrigel invasion chambers. Invaded cells in four fields per group were counted. Data are representative of three independent experiments. Columns, mean; bars, SD. P-value as compared to non-treated scramble-control cells. (E) Confluent control and BART RNAi S2-013 cells were preloaded with or without C3 exoenzyme and wounded. The dashed lines indicate the border of the scratch (time 0), made using a plastic pipette tip. The number of cells which migrated into the initially cell-free scratch was counted. Cells in four defined areas per group were quantified for each experiment. Data are representative of three independent experiments. Columns, mean; bars, SD. P-value as compared to non-treated scramble-control cells.

localization of endogenous BART and ARL2 in S2-013 cells by immunofluorescence (Fig. 1B). Staining of BART and ARL2 colocalized in the cytoplasm.

To determine the effect of BART depletion on the localization of ARL2, plasmids expressing small interfering RNAs (siRNAs) specific for BART were stably expressed in S2-013 cells (Fig. 1C). A wound-healing assay was used to observe the localization of BART and ARL2 in polarized migrating cells (Fig. 1D). We confirmed that knockdown of BART in S2-013 and PANC-1 enhanced cell motility into a wounded area of confluent cultures compared to control cells (10). BART and ARL2 were both recruited to the leading edges during wound healing of control S2-013 cells (upper panels in Fig. 1D).
However, depletion of BART inhibited the accumulation of ARL2 at the leading edges (lower panels in Fig. 1D). These results indicate that BART and ARL2 are associated with cell migration and that ARL2 localizes at the leading edges in a BART-dependent manner in migrating PDAC cells.

ARL2 decreases the level of active RhoA. We examined the effects of ARL2 on the activity of small G-protein members of the Rho family Racl and RhoA, which are important regulators of both actin dynamics and cell-substratum adhesions in migratory cells. Rho family small GTPases are central regulators of actin-based cell motility (13). The myc-tagged wild-type ARL2 and its point mutated forms, Q70L and T30N, were transiently 
A

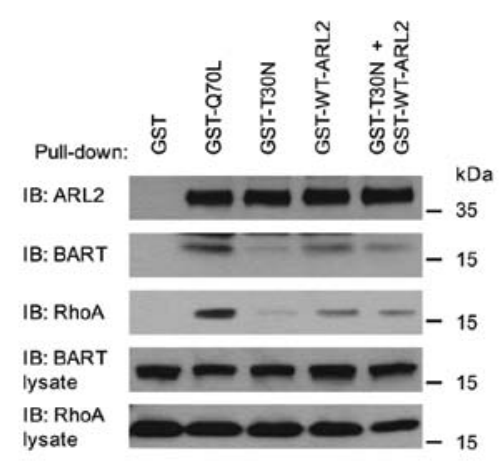

B
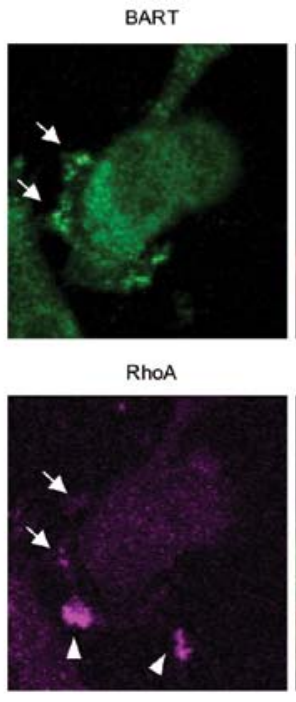

RhoA

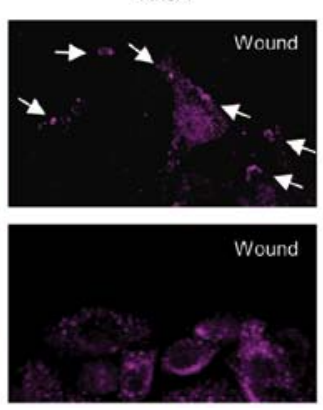

ARL2

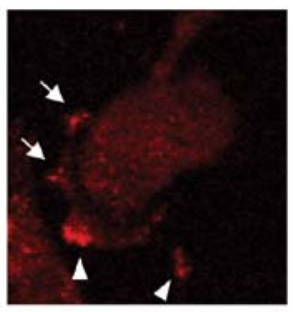

Merge/DAPI

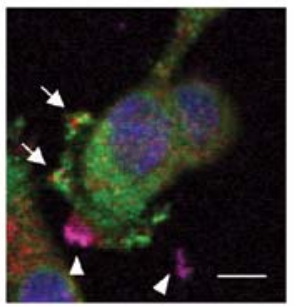

C
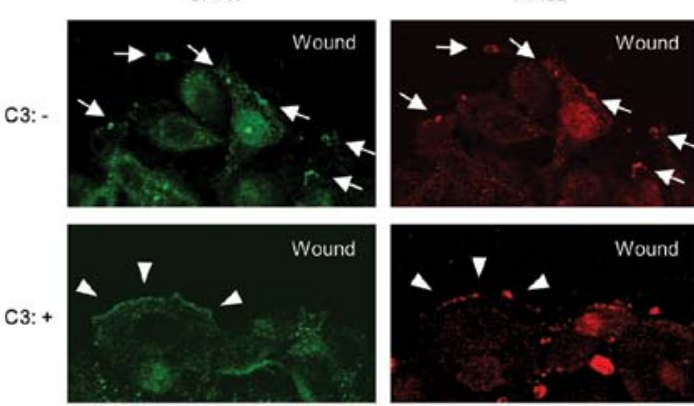

Figure 3. GTP-ARL2 binds to BART and active RhoA at cell protrusions in PDAC cells. (A) GST-bound wild-type and mutant ARL2 proteins were used for affinity precipitation in GST-pull-down assays. Equal amounts of S2-013 cell lysates were incubated with $8 \mu \mathrm{g}$ of the GST-fusion protein, and the precipitated BART and RhoA were detected by Western blotting. (B) Immunocytochemical staining of S2-013 cells, using anti-BART (green), anti-ARL2 (red) and antiRhoA (violet) antibodies. Blue, DAPI staining. Arrowheads, colocalized ARL2 and RhoA; arrows, colocalized BART, ARL2 and RhoA in lamellipodial-like protrusions. Bar, $10 \mu \mathrm{m}$. (C) S2-013 cells were preloaded with or without C3 exoenzyme, and immunocytochemical staining was performed, using anti-BART (green), anti-ARL2 (red) and anti-RhoA (violet) antibodies. Blue, DAPI staining. Arrows, colocalized BART, ARL2 and RhoA at the leading edge; arrowheads, colocalized BART and ARL2 at the leading edge. Bars, $10 \mu \mathrm{m}$.

transfected into S2-013 cells, followed by a GST-pull-down assay using GST-PAK-CRIB or GST-Rhotekin to estimate the level of active Rac1 and RhoA, respectively (Fig. 2A). The ARL2-Q70L mutant is GTPase defective (GTP-bound type), and the ARL2-T30N mutant is defective in GTP binding (GDP-bound type) (11). We found that, like wild-type ARL2, the GTP-bound form of ARL2-Q70L, decreased the amount of active RhoA, but it did not alter the amount of active Rac1. We infer from this result that, to prevent RhoA activation, ARL2 must be in the GTP-bound form. On the other hand, silencing of BART by siRNA in human PDAC S2-013 and PANC-1 cells caused a decrease in the level of active RhoA (Fig. 2B), suggesting that endogenous BART is associated with an increase in the level of active RhoA. These results suggest the possibility that the inactivation of RhoA by ARL2 might be inhibited by binding of BART to ARL2.

RhoA has been implicated in the regulation of directed cell migration (14). We next questioned whether RhoA influences PDAC cell migration and the potential role of BART in RhoA-mediated migration, by analyzing cell invasion of control and BART RNAi S2-013 cells after cell loading with the Rho inhibitor C3 exoenzyme. We first confirmed that loading of control S2-013 cells with the C3 exoenzyme decreased the level of GTP-RhoA (Fig. 2C). We previously reported that suppression of BART enhanced cell invasiveness in Matrigel invasion assays (10). Pretreatment of control S2-013 cells with C3 induced the same level of invasive activity in the Matrigel assay as that induced by knocking down BART (Fig. 2D). We also confirmed that C3 treatment of S2-013 cells enhanced cell motility into a wounded area of confluent cultures to the same level as that resulting from BART knockdown (Fig. 2E). Based on these results, we hypothesized that BART-dependent inhibition of PDAC cell invasion is likely to be associated with active RhoA via inhibition of GTP-ARL2 function.

BART is required for the binding of ARL2 to RhoA. Pull-down techniques were used to confirm the interaction between GST-tagged ARL2 (wild-type and its mutated forms, Q70L and T30N) and endogenous BART and RhoA in S2-013 cells (Fig. 3A). The binding of GST-ARL2-Q70L to BART and RhoA was enhanced compared to that of GST-wild-type ARL2, whereas the binding of GST-ARL2-T30N to BART and RhoA was significantly decreased. It is noteworthy that GST-pulldown using both GST-wild-type ARL2 and GST-ARL2-T30N 
A

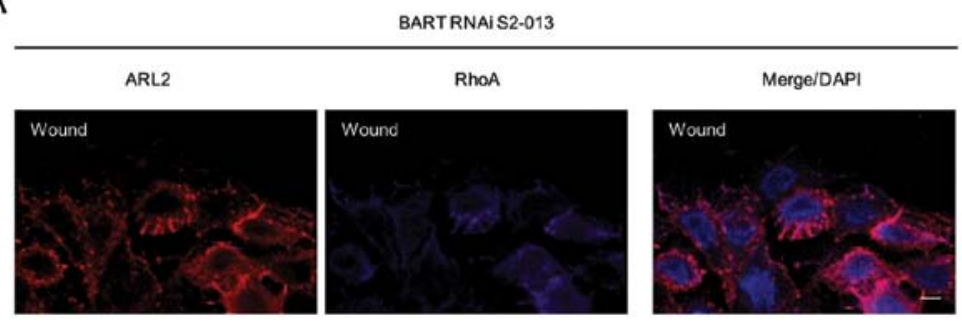

B
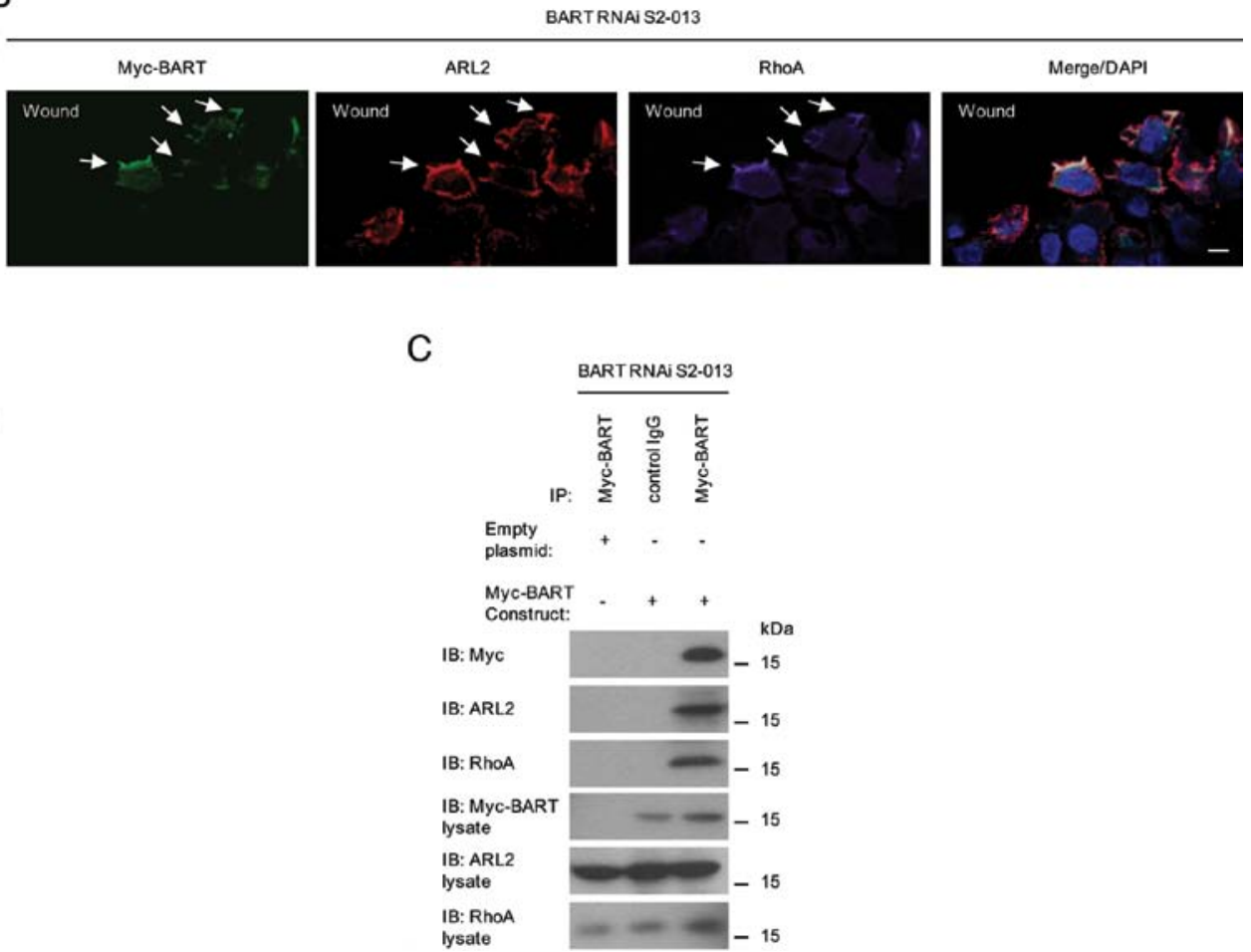

Figure 4. BART is required for the interaction of ARL2 with RhoA. (A) Confluent BART RNAi S2-013 cells (siBART-1) were wounded. After 4 h, cells were immunostained with anti-ARL2 (red) and anti-RhoA (violet) antibodies. Blue, DAPI staining. Bar, $10 \mu \mathrm{m}$. (B) To identify the effects of rescuing BART expression on the colocalization of ARL2 and RhoA, myc-tagged BART was transfected into BART RNAi S2-013 cells (siBART-1). After 48 h, cells were wounded and immunostained with anti-myc (green), anti-ARL2 (red) and anti-RhoA (violet) antibodies. Blue, DAPI staining. Arrows, colocalized myc-tagged BART, endogenous ARL2 and RhoA at the leading edge. Bar, $10 \mu \mathrm{m}$. (C) Immunoprecipitation of exogenous BART from BART RNAi S2-013 cells. The myc-tagged BART construct was transiently transfected and immunoprecipitated using anti-myc antibody. Immunoprecipitates were examined by Western blotting using anti-myc, anti-ARL2 and anti-RhoA antibodies. Rabbit IgG was used as an isotype control.

resulted in a significant increase in the amount of co-precipitated BART and RhoA compared to pull-down of GST-ARL2-T30N alone. These results suggest that the active form of ARL2 strongly binds to both BART and RhoA.

Immunostaining of S2-013 cells showed that ARL2 colocalized with RhoA in lamellipodial-like protrusions (arrowheads in Fig. 3B). Dramatic actin cytoskeletal reorganization, membrane ruffling and the formation of membrane lamellipodial/filopodial protrusions are associated with cell migration (15). Of note, BART was also partially colocalized with ARL2 and RhoA in the membrane protrusions (arrows in Fig. 3B). To determine if BART and ARL2 bind to active RhoA at the leading edge, monolayers of S2-013 cells were scratch-wounded to induce polarized cell migration. These cells were preloaded or not with $\mathrm{C} 3$, and the localization of endogenous BART, ARL2 and RhoA was determined using immunofluorescence (Fig. 3C). Significant amounts of BART, ARL2 and RhoA were recruited to, and co-localized at the leading edges during wound healing of S2-013 cells without C3 treatment (arrows in Fig. 3C), whereas C3 loading inhibited the recruitment of RhoA to BART and ARL2 colocalized at the leading edges. Combined with the result of Fig. 3A, these results support the finding that BART interacts with the active forms of ARL2 and RhoA at the leading edges.

We next examined the effects of depleting BART on the localization of ARL2 and RhoA at the wound edge of migrating cells. We found that depletion of BART inhibited the accumulation and colocalization of ARL2 and RhoA at the leading edges (Fig. 4A) but that a small population of ARL2 and RhoA colocalized in the cytoplasm of BART RNAi S2-013 cells. We also overexpressed myc-tagged BART in BART RNAi S2-013 cells to establish whether BART is sufficient for facilitating the binding of ARL2 with RhoA in this system (Fig. 4B). Exogenous overexpressed myc-BART restored the colocalization of ARL2 and RhoA at the leading edges. Complex formation of exogenous myc-BART and endogenous 

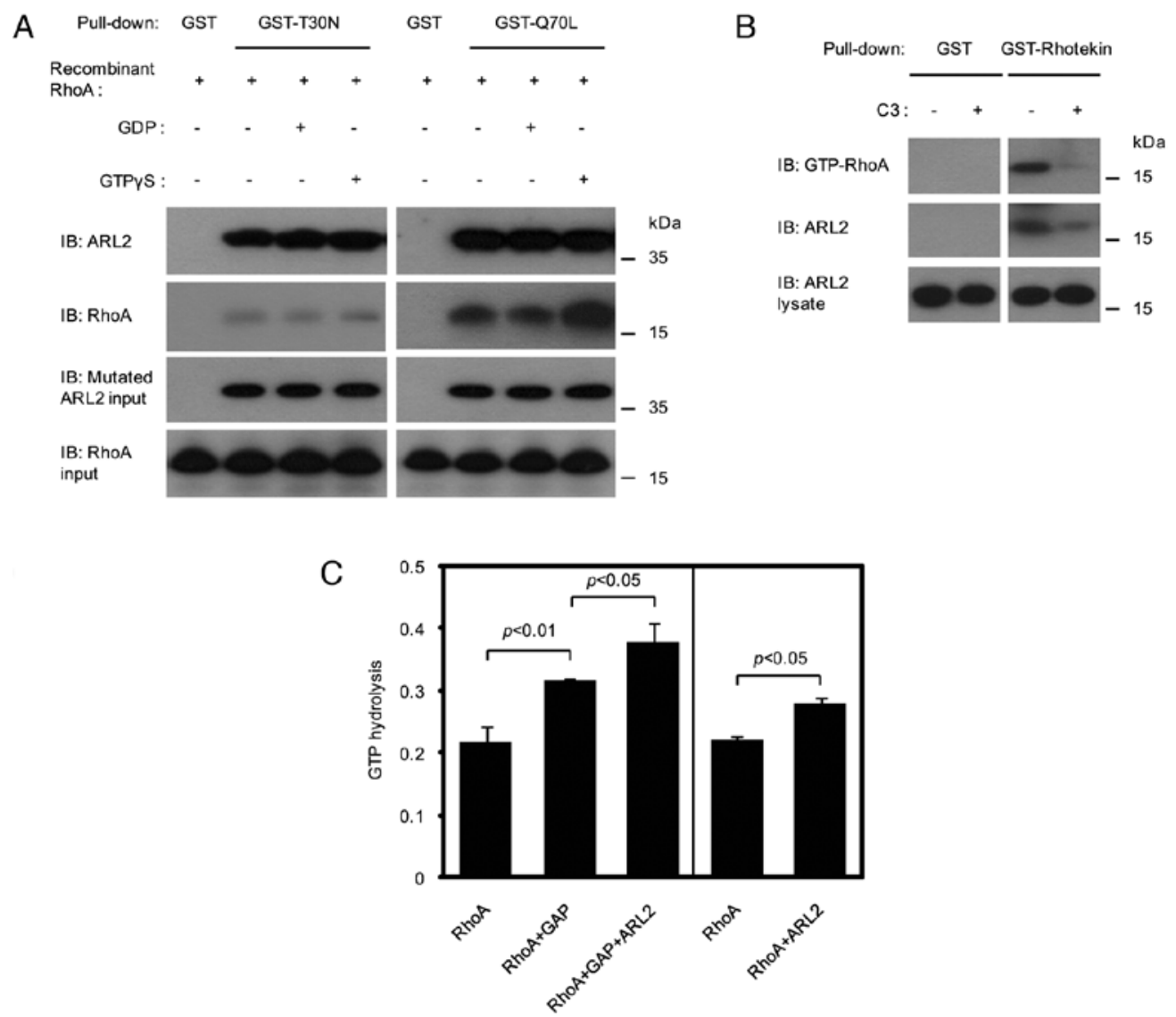

Figure 5. GTP-ARL2 interacts with GTP-RhoA and inactivates RhoA. (A) Incubation of recombinant RhoA with GDP or GTP $\gamma \mathrm{S}$ was followed by pull-down experiments using GST-ARL2-Q70L or GST-ARL2-T30N. Precipitates were examined by Western blotting using anti-RhoA antibody. Data are representative of three independent experiments. (B) S2-013 cells were preloaded with or without C3 exoenzyme, and equal amounts of cell lysates were precipitated with GSTRhotekin, followed by Western blotting with anti-ARL2 and anti-RhoA antibodies. Data are representative of three independent experiments. (C) To determine if wild-type ARL2 has a GAP function for RhoA GTPase, we carried out in vitro GAP assays using recombinant ARL2 protein produced in Escherichia coli. Data are representative of three independent experiments and are shown as means \pm SEM.

ARL2 and RhoA was confirmed by immunoprecipitation with an anti-myc antibody (Fig. 4C). These results suggest that BART is necessary for the interaction and localization of active forms of ARL2 and RhoA at the front of the migrating cancer cells.

The active form of ARL2 binds to GTP-bound RhoA and inactivates RhoA. We used a second method to detect specific interaction between GTP-bound RhoA and the active form of ARL2. Recombinant RhoA that was incubated with GDP or GTP $\gamma \mathrm{S}$ was used in pull-down experiments with GST-tagged ARL2-Q70L or ARL2-T30N (Fig. 5A). The amount of GTP-RhoA that bound to GST-ARL2-Q70L was significantly higher than that bound to GST-ARL2-T30N, which only weakly interacted with GTP-RhoA. Furthermore, pull-down assays of S2-013 cells using GST-Rhotekin, which binds to GTP-RhoA, pulled down both ARL2 and active RhoA. However, the level of ARL2 in the pull-downs decreased when C3 exoenzyme loaded cells were used, indicating that endogenous ARL2 interacts with active RhoA (Fig. 5B). These results confirmed that GTP-bound RhoA preferentially interacts with the active form of ARL2 in PDAC cells.

We hypothesized that RhoA might be a direct substrate of ARL2. Rho GTPase-activating proteins (RhoGAPs) stimulate the low intrinsic GTPase activity of Rho GTPase proteins, leading to conversion of GTP-bound active forms of Rho GTPases to GDP-bound inactive forms. In order to determine if wild-type ARL2 has GAP function, we performed in vitro GAP assays using recombinant wild-type ARL2 protein (Fig. 5C). We incubated recombinant ARL2 and human RhoA GTPase with or without the human p50 RhoGAP protein. The GAP domain from p50 RhoGAP stimulates GTPase activities of RhoA in vitro. In these studies, ARL2 also markedly increased the GTPase activity of RhoA (Fig. 5C). In addition, it is likely that ARL2 increased the GTPase activity of p50 RhoGAP towards RhoA (Fig. 5C). These results suggest that RhoA is the preferred substrate of ARL2 GAP activity.

BART inhibits the function of ARL2 in actin-cytoskeletal rearrangements. The Rho family constituting Rac1, Cdc42 and RhoA are important regulators of actin dynamics and cellsubstratum adhesions in migratory cells, and thus are critically involved in cell motility and invasion (16-18). Moreover, active RhoA has been shown to be required for cadherin clustering in fibroblasts, suggesting that RhoA regulates cadherindependent cell adhesion (17). Phalloidin staining of F-actin showed that S2-013 cells transfected with myc-ARL2-Q70L extended many peripheral actin-rich structures in membrane 

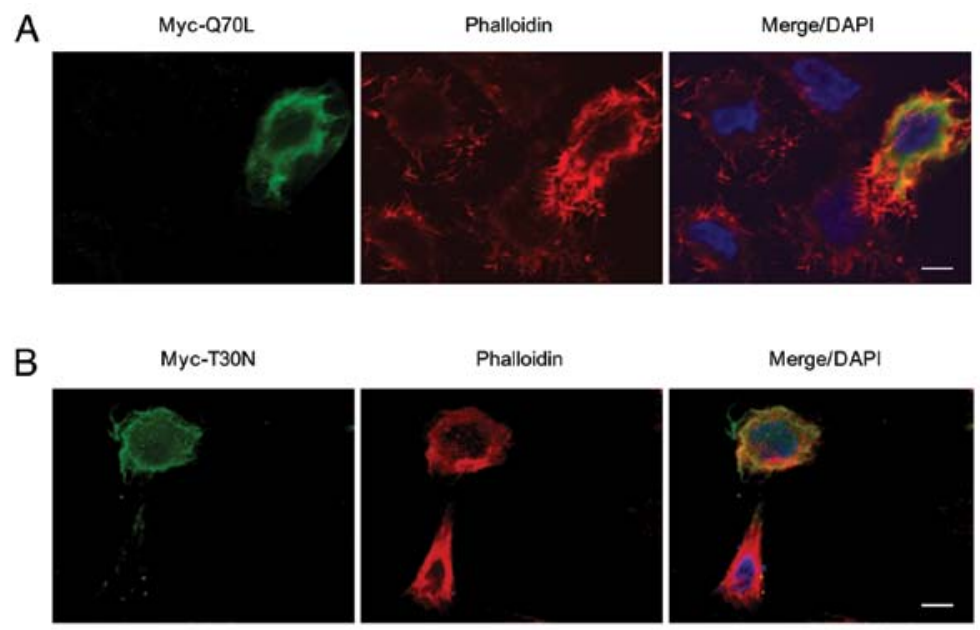

C

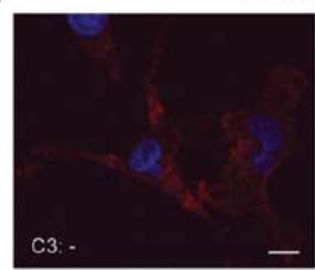

Phalloidin/DAP

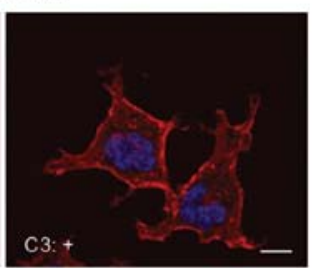

BARTRNAIS2-013

E
D

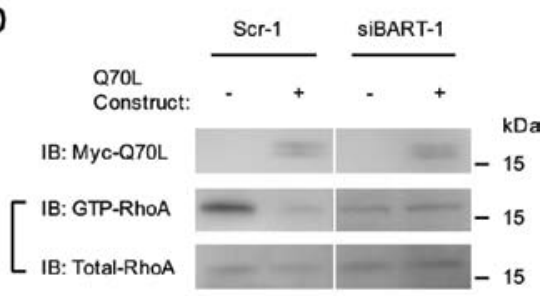

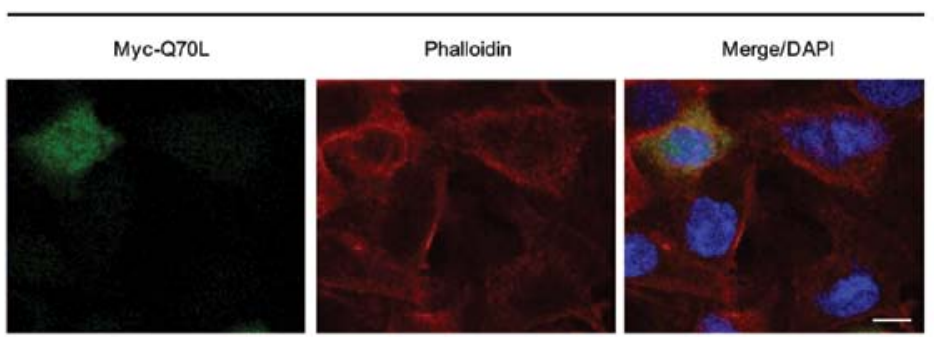

Figure 6. GTP-ARL2 induces actin-cytoskeleton rearrangements and BART inhibits the formation of an actin meshwork at protrusions through inhibition of ARL2 activity. (A) Immunocytochemical staining was performed $48 \mathrm{~h}$ after transfection of myc-tagged ARL2-Q70L into S2-013 cells using anti-myc antibody (green). The actin cytoskeleton (red) was stained with phalloidin. Blue, DAPI staining. Bar, $10 \mu \mathrm{m}$. (B) Immunocytochemical staining was performed $48 \mathrm{~h}$ after transfection of myc-tagged ARL2-T30N into S2-013 cells, using anti-myc antibody (green). Actin (red) was stained with phalloidin. Blue, DAPI staining. Bar, $10 \mu \mathrm{m}$. (C) S2-013 cells were preloaded with or without C3 exoenzyme and phalloidin staining was performed (red). Blue, DAPI staining. Bars, $10 \mu \mathrm{m}$. (D) The amount of active GTP-loaded RhoA was determined $48 \mathrm{~h}$ after transfection of myc-tagged ARL2-Q70L into control (Scr-1) and BART RNAi (siBART-1) cells, by GST-pull-down assays using GST-Rhotekin. GTP-RhoA was detected by Western blotting. (E) Immunocytochemical staining was performed 48 h after transfection of myc-tagged ARL2-Q70L into BART RNAi S2-013 cells (siBART-1) using anti-myc antibody (green). Actin was stained with phalloidin. Blue, DAPI staining. Bar, $10 \mu \mathrm{m}$.

ruffles at the edge of cell protrusions (Fig. 6A). Phalloidin staining of S2-013 cells transfected with myc-ARL2-T30N was similar to that of untransfected S2-013 cells (Fig. 6B). The myc-ARL2-T30N-expressing cells did not induce peripheral actin-cytoskeletal rearrangements. We confirmed that the C3 exoenzyme induced peripheral actin bundling in S2-013 cells (Fig. 6C). As seen in Fig. 2D and E, C3 loading induces invasive activity of PDAC cells. Thus, it is possible that RhoA inhibits cell invasion through the inhibition of peripheral actin-cytoskeletal rearrangements and that ARL2 plays an important role in regulating cell invasion. ARL2-Q70L is presumably primarily GTP-bound and inhibits activation of RhoA. Therefore, in order to induce peripheral actin-cytoskeletal rearrangements, ARL2 must be GTP-bound.
The overexpression of exogenous myc-tagged ARL2-Q70L in BART RNAi S2-013 cells did not induce any significant change in RhoA activity (Fig. 6D). This result indicates that BART modulates the function of GTP-bound ARL2 in RhoA inactivation. To investigate the role of BART and GTP-bound ARL2 in peripheral actin-cytoskeletal rearrangements, myctagged ARL2-Q70L was transfected into BART RNAi S2-013 cells (Fig. 6E). Overexpression of the exogenous myc-ARL2Q70L mutant in BART RNAi cells had no obvious effect on the rearrangement of the actin-cytoskeleton. Thus, BART plays a role in preventing peripheral actin-cytoskeletal rearrangements caused by the expression of myc-ARL2-Q70L. These data reinforce our conclusion that BART increases the level of active RhoA, inhibits subsequent actin-cytoskeletal rearrangements by 
inhibiting the function of GTP-bound ARL2 and thereby plays a role in inhibiting cell invasion.

\section{Discussion}

PDAC is among the most fatal of cancers due to its ability to extensively invade surrounding tissues and metastasize at an early stage (19). Extensive local infiltration and metastasis are the main causes of death in PDAC (20). Here, we examined the functional roles of BART and ARL2 in regulating cell migration in PDAC.

BART is a protein that binds ARL2, which belongs to the ARL family of proteins (1). In this study, we show that BART and ARL2 colocalize in the cytoplasm and accumulate at leading edges of PDAC cells. We found that depletion of BART diminished the accumulation of ARL2 at leading edges (Fig. 1D), indicating that BART is required for the recruitment of ARL2 to leading edges and that both BART and ARL2 are necessary for regulating the migration of PDAC cells.

To investigate the roles of ARL2 in modulation of the activity of Rho proteins, which are important regulators of both actin dynamics and cell-substratum adhesions in migratory cells, we performed GST pull-down assays using Racl- and RhoA-binding domains. Experiments using GTPase-defective and GTP-binding-defective mutants of ARL2 revealed that GTP-ARL2 binds with active RhoA (Figs. 3A, 5A and B) and that it is the GTP-bound form of ARL2 that preferentially inhibits activation of RhoA (Fig. 2A). In contrast, when BART expression was suppressed, we found that the level of active RhoA was decreased (Fig. 2B), thus indicating that endogenous BART functions to positively regulate RhoA activity. The result that GTP-bound ARL2 is sufficient for decreasing the level of active RhoA in this system suggests that BART is likely to inhibit the function of GTP-ARL2 by directly binding to ARL2.

BART colocalizes with active forms of ARL2 and RhoA at leading edges of migrating PDAC cells (Fig. 3A and C). Knocking down of Smurf1, a HECT domain E3 ubiquitin ligase, induces colocalization of RhoA and F-actin in membrane protrusions and suppresses the tumorigenic morphology and motility of HEK293T cells (21). This result suggests that RhoA signaling is associated with dynamic membrane movement and inhibits cell motility. Depletion of BART diminished the accumulation of ARL2 and RhoA at leading edges (Fig. 4A), and their accumulation at leading edges was restored by the expression of myc-tagged BART (Fig. 4B). Preloading of control cells with the Rho inhibitor $\mathrm{C} 3$ exoenzyme induces invasive activity in Matrigel and wound-healing assays as efficiently as BART RNAi (Fig. 2D and E). Thus, decreased activity of RhoA induces cell invasion, and active RhoA is required for the anti-invasive activity of BART. On the basis of these results, it is most likely that BART is required for the binding of GTP-ARL2 to active RhoA at leading edges and that BART prevents GTP-ARL2 inhibition of RhoA and thereby inhibits cell invasion of PDAC cells. Further studies are needed to demonstrate the mechanism by which the trimeric BART/ARL2/RhoA complex is formed at the leading edge and by which direct binding of BART to ARL2 inhibits ARL2 activity.

The migratory competence of tumor cells requires activation of the motile cycle, the first step of which is actin remodeling, which drives the formation of cell protrusions, defines the direc- tion of migration and initiates the growth of the lamellipodium (22). Dynamic actin remodeling processes at the leading edge of migrating cells are complex and involve increased actin filament severing, capping and dendritic branching (23). The concerted regulation of these events is mediated by a complex temporal and spatial interplay of Rho GTPases, kinases and phosphatases (22). Rho GTPases and their effectors are key intracellular signaling molecules that coordinate the cytoskeletal remodeling that is required for cell spreading, motility and cell-shape changes (24). The results of our immunofluorescence studies show that myc-ARL2-Q70L overexpression induces surface rearrangements of the actin cytoskeleton (Fig. 6A). Dynamic, actin-based plasma membrane protrusions that control growth cone path finding include lamellipodia, in which the actin cytoskeleton assumes a crosslinked and branched meshwork, and filopodia, which consist of parallel bundles of actin filaments protruding from the growth cone or lamellipodial margin (25). This result indicates that GTP-bound ARL2 is a physiological trigger that elicits redistribution of actin leading to peripheral actin-rearrangements. Interestingly, myc-ARL2-Q70L did not change the level of active RhoA in BART RNAi S2-013 cells (Fig. 6D). Additionally, peripheral actin-rearrangements were not induced when myc-ARL2-Q70L was overexpressed in BART RNAi S2-013 cells (Fig. 6E). Membrane ruffles and protrusions are characteristically present at the leading edge of motile cells, whereas stress fibers that promote adhesion to the substratum correlate negatively with cell locomotion (26). Inactivation of Rho kinase, by the Rho kinase inhibitor Y-27632 (27), leads to the dissolution of stress fibers and enhances cell movement during wound healing (15). Given the effect of the BART and ARL2 complex on peripheral actin-rearrangements, this complex must be associated with the regulation of membrane ruffles but not stress fibers, thereby allowing BART to inhibit cell invasion of PDAC cells through ARL2.

The findings presented in this study are supportive of the pivotal roles of BART and ARL2 in the coordinated regulation of cortical actin changes via the regulation of RhoA activity. We have established the functional significance of the interaction of BART with ARL2 in inhibition of the invasiveness of PDAC cells.

\section{Acknowledgements}

We thank Michael Hollingsworth for many helpful experimental suggestions. We also thank Keith Johnson for kindly providing us with the pGEX-Rhotekin and -PAK-CRIB plasmids, Nicholas Cowan for providing the pET23b-ARL2Q70L and ARL2-T30N plasmids and Aki Tanouchi for her excellent technical assistance. This study was supported by a Grant-in-Aid from the Ministry of Health, Labour and Welfare of Japan (to T.S.).

\section{References}

1. Sharer JD and Kahn RA: The ARF-like 2 (ARL2)-binding protein, BART. Purification, cloning, and initial characterization. J Biol Chem 274: 27553-27561, 1999.

2. Clark J, Moore L, Krasinskas A, Way J, Battey J, Tamkun J and Kahn RA: Selective amplification of additional members of the ADP-ribosylation factor (ARF) family: cloning of additional human and Drosophila ARF-like genes. Proc Natl Acad Sci USA 90: 8952-8956, 1993. 
3. Zhou C, Cunningham L, Marcus AI, Li Y and Kahn RA: Arl2 and Arl3 regulate different microtubule-dependent processes. Mol Biol Cell 17: 2476-2487, 2006.

4. Bailey LK, Campbell LJ, Evetts KA, Littlefield K, Rajendra E, Nietlispach D, Owen D and Mott HR: The structure of binder of Arl2 (BART) reveals a novel $G$ protein binding domain: implications for function. J Biol Chem 284: 992-999, 2008.

5. Zhang T, Li S, Zhang Y, Zhong C, Lai Z and Ding J: Crystal structure of the ARL2-GTP-BART complex reveals a nove recognition and binding mode of small GTPase with effector Structure 17: 602-610, 2009.

6. Muromoto R, Sekine Y, Imoto S, Ikeda O, Okayama T, Sato N and Matsuda T: BART is essential for nuclear retention of STAT3. Int Immunol 20: 395-403, 2008

7. Aznar S, Valerón PF, Del Rincon SV, Pérez LF, Perona R and Lacal JC: Simultaneous tyrosine and serine phosphorylation of STAT3 transcription factor is involved in Rho A GTPase oncogenic transformation. Mol Biol Cell 12: 3282-3294, 2001.

8. Debidda M, Wang L, Zang H, Poli V and Zheng Y: A role of STAT3 in Rho GTPase-regulated cell migration and proliferation. J Biol Chem 280: 17275-17285, 2005.

9. Schmitz AA, Govek EE, Böttner B and van Aelst L: Rho GTPases: signaling, migration, and invasion. Exp Cell Res 261: $1-12,2000$.

10. Taniuchi K, Nishimori I and Hollingsworth MA: Intracellular CD24 inhibits cell invasion by post-transcriptional regulation of BART through interaction with G3BP. Cancer Res 71: 895-905, 2011.

11. Bhamidipati A, Lewis SA and Cowan NJ: ADP ribosylation factor-like protein 2 (Arl2) regulates the interaction of tubulinfolding cofactor D with native tubulin. J Cell Biol 149: 1087-1096, 2000.

12. Colinet D, Schmitz A, Depoix D, Crochard D and Poirié M: Convergent use of RhoGAP toxins by eukaryotic parasites and bacterial pathogens. PLoS Pathog 3: e203, 2007.

13. Hall A: Rho GTPases and the actin cytoskeleton. Science 279 509-514, 1998.

14. Maekawa M, Ishizaki T, Boku S, Watanabe N, Fujita A, Iwamatsu A, Obinata T, Ohashi K, Mizuno K and Narumiya S: Signaling from Rho to the actin cytoskeleton through protein kinases ROCK and LIM-kinase. Science 285: 895-898, 1999.
15. Nobes CD and Hall A: Rho GTPases control polarity, protrusion, and adhesion during cell movement. J Cell Biol 144: 1235-1244, 1999.

16. Nobes CD and Hall A: Rho, Rac, and Cdc42 GTPases regulate the assembly of multimolecular focal complexes associated with actin stress fibers, lamellipodia, and filopodia. Cell 81: 53-62, 1995.

17. Takaishi K, Sasaki T, Kotani H, Nishioka $H$ and Takai $Y$ : Regulation of cell-cell adhesion by rac and rho small $\mathrm{G}$ proteins in MDCK cells. J Cell Biol 139: 1047-1059, 1997.

18. Etienne-Manneville S and Hall A: Rho GTPases in cell biology. Nature 420: 629-635, 2002

19. Baumgart M, Heinmöller E, Horstmann O, Becker H and Ghadimi BM: The genetic basis of sporadic pancreatic cancer. Cell Oncol 27: 3-13,2005.

20. Ahrendt SA and Pitt HA: Surgical management of pancreatic cancer. Oncology 16: 725-734, 2002.

21. Wang HR, Zhang Y, Ozdamar B, Ogunjimi AA, Alexandrova E, Thomsen GH and Wrana JL: Regulation of cell polarity and protrusion formation by targeting RhoA for degradation. Science 302: 1775-1779, 2003.

22. Eiseler T, Döppler H, Yan IK, Kitatani K, Mizuno K and Storz P: Protein kinase D1 regulates cofilin-mediated $\mathrm{F}$-actin reorganization and cell motility through slingshot. Nat Cell Biol 11: 545-556, 2009.

23. Bamburg JR, McGough A and Ono S: Putting a new twist on actin: ADF/cofilins modulate actin dynamics. Trends Cell Biol 9: 364-370, 1999.

24. Ridley AJ: Rho GTPases and cell migration. J Cell Sci 114: 2713-2722, 2001.

25. Gallo G and Letourneau PC: Regulation of growth cone actin filaments by guidance cues. J Neurobiol 58: 92-102, 2004.

26. Boshans RL, Szanto S, van Aelst L and D'Souza-Schorey C: ADP-ribosylation factor 6 regulates actin cytoskeleton remodeling in coordination with Rac1 and RhoA. Mol Cell Biol 20 3685-3694, 2000.

27. Uehata M, Ishizaki T, Satoh H, Ono T, Kawahara T, Morishita T, Tamakawa H, Yamagami K, Inui J, Maekawa M and Narumiya S: Calcium sensitization of smooth muscle mediated by a Rho associated protein kinase in hypertension. Nature 389: 990-994, 1997. 